\title{
Changes in the Anterior Lens Epithelium and Basement Membrane in Pseudoexfoliation Syndrome undergoing Surgery for Senile Cataracts: A Transmission Electron Microscopic Study
}

\author{
Ozlem Dikmetas ${ }^{1}$, Yasemin Kapucu ${ }^{1}$, Aysegul Firat ${ }^{2}$, Mustafa Fevzi Sargon ${ }^{3}$ and Sibel Kocabeyoglu ${ }^{1}$ \\ ${ }^{1}$ Department of Ophthalmology, Hacettepe University School of Medicine, Ankara, Turkey \\ ${ }^{2}$ Department of Anatomy, Hacettepe University School of Medicine, Ankara, Turkey \\ ${ }^{3}$ Department of Anatomy, Lokman Hekim University School of Medicine, Ankara, Turkey
}

\begin{abstract}
Objective: To compare the electron microscopic lens findings with and without pseudoexfoliation syndrome (XFS), in patients undergoing surgery for senile cataract.

Study Design: A descriptive study.

Place and Duration of the Study: Ophthalmology Department, Hacettepe University Hospital between December 2019 and March 2020.

Methodology: Anterior lens capsules (basement membrane and lens epithelial cells) taken from 20 eyes of 20 patients with senile cataract were examined. XFS was detected by slit-lamp examination in 10 of 20 patients. Remaining 10 were included in the control group. All patients and controls were of similar age and had no systemic disease. The anterior lens capsules were obtained from cataract surgery and prepared for observation under a transmission electron microscope.

Results: The mean age of individuals in the XFS and control groups was $69.4 \pm 6.9$ (56-82) years and $65.2 \pm 6.9$ (54-73) years, respectively. The best-corrected visual acuity in the XFS and control groups was $0.7 \pm 0.4(0.3-1.3) \log M A R$ and $0.9 \pm$ $0.4(0.4-1.3) \log M A R$, respectively. Marked ultrastructural changes were observed in the anterior lens capsules of all eyes with pseudoexfoliation. Degenerative changes and thinning in lens epithelial cells were observed in all samples. The lens epithelial cells were loosely attached to the basement membrane in places. Numerous apoptotic cells with pyknotic nuclei were observed in lens epithelial cells, and many vacuoles within the cytoplasm in different areas were detected.

Conclusion: Transmission electron microscopic examination revealed ultrastructural abnormalities in the XFS epithelium of all patients in the XFS group. Many aspects of the pathogenetic process of XFS remain uncertain, hence requiring further exploration.
\end{abstract}

Key Words: Capsule, Electron microscopy, Epithelium, Pseudoexfoliation syndrome.

How to cite this article: Dikmetas O, Kapucu Y, Firat A, Sargon MF, Kocabeyoglu S. Changes in the Anterior Lens Epithelium and Basement Membrane in Pseudoexfoliation Syndrome undergoing Surgery for Senile Cataracts: A Transmission Electron Microscopic Study. J Coll Physicians Surg Pak 2021; 31(11):1346-1350.

\section{INTRODUCTION}

Pseudoexfoliation syndrome (XFS) is recognised as one of the mostcommon identifiable causes of glaucoma worldwide. Accumulation of white deposits of exfoliative material on the anterior lens capsule (ALC) is the most characteristic clinical feature of XFS. ${ }^{1}$

Correspondence to: Dr. Ozlem Dikmetas, Department of Ophthalmology, Hacettepe University School of Medicine, Ankara, Turkey

E-mail: ozlemdikmetas@gmail.com

Received: November 02, 2020; Revised: March 06, 2021;

Accepted: March 16, 2021

DOI: https://doi.org/10.29271/jcpsp.2021.11.1346
Senile cataracts are among the most common causes of the reversible loss of vision worldwide; the prevalence and severity of cataracts increase with aging. ${ }^{2}$ Investigators have concluded that a relationship exists between cataract formation and functional/structural organisation disorder of the lens epithelium. ${ }^{3}$ Degenerative changes and laminin-positive linear capsular deposits appear with aging. ${ }^{4} \mathrm{~A}$ limited number of transmission electron microscopy (TEM) studies have been conducted on lens structure. ${ }^{5}$ Morphological studies detected structural changes in the lens epithelium and capsule in senile cataracts; ${ }^{5}$ however, no significant change in anterior capsular thickness was detected. ${ }^{5}$ Ultrastructural studies of the lens revealed exfoliative material deposits on the surface of the basement membrane of the anterior capsule; whereas, average lens thickness and elasticity were similar compared with those in the 
control group. ${ }^{6}$ The histopathological studies demonstrated the active production of a pathological capsular substance in the epithelial cells in the germinative zone of the lens in eyes with XFS.After five years of examination in 46 eyes, Puska et al. identified pseudoexfoliation as a risk factor for cataract formation. ${ }^{7}$

Pseudoexfoliation syndrome is an age-related and progressive disease affecting all anterior segment structures by accumulating fibrillar extracellular materials. As the number of studies made to clarify the actual effect of XFS on lens structures is limited, it is needed to examine lenticular structures under the electron microscope and define the structure and the origin of the exfoliative material in detail.

The aim of this study was to determine the capsular and epithelial changes in eyes with XFS, compared with the control group; and examine the histopathology of the exfoliative material using TEM.

\section{METHODOLOGY}

This descriptive study was conducted at the Ophthalmology Department, Hacettepe University School of Medicine between December 2019 and March 2020. All patients underwent routine uneventful phacoemulsification and intraocular lens implantation surgery. Informed consent was obtained from all the patients before the surgery. The study followed the tenets of the Declaration of Helsinki and was approved by the Ethics Committee of the University.

All patients underwent routine ophthalmological examination, including detection of best-corrected visual acuity, slit-lamp examination, and dilated fundus examination. Exfoliative material on the anterior lens surface and the presence of cataracts were detected by the slit-lamp examination (Figure 1). In both XFS and the control groups, intraocular pressure measurements were within the normal range. Besides, any of the 20 patients included in the study had glaucoma. Only nuclear cataracts and cataracts with grade 3 or below were included in the study. Three additional exclusion criteria were as follows: having systemic or ocular diseases including glaucoma other than senile cataracts, taking any ocular treatments, and having a history of trauma. The patients underwentroutine phacoemulsification and intraocular lens implantation surgery by the same surgeon (SK) under local anesthesia. Routine anterior capsulorrhexis, lens aspiration, and intraocular lens implantation were performed. A 5- to 5.5-mm circular portion of the central anterior lens capsule (ALC) was carefully removed and immediately fixed in a solution of neutral-buffered $2.5 \%$ glutaraldehyde for TEM. The ALCs were put into $2.5 \%$ glutaraldehyde for 24 hours for primary fixation. After fixation, they were washed with the same buffer and dehydrated in increasing concentrations of alcohol series. After dehydration, the tissues were washed with propylene oxide and embedded in epoxy resin embedding media. The semi-thin and ultrathin sections of the obtained tissue blocks were cut using an ultramicrotome (LKB Nova, Sweden). These 2- $\mu$ m-thick semithin sections were stained with methylene blue and examined under a light microscope (Nikon, Japan). Afterward, the tissue blocks weretrimmed, and ultrathin sections of about 60 -nm thick- ness were taken using the same ultramicrotome. These ultrathin sections were stained with uranyl acetate and lead citrate and examined undera JeolJEM 1200 EX (Japan) transmission electron microscope. All data were analysed using the SPSS software (IBM SPSS 17; SPSS Inc., USA) and summarized as mean and standard deviations.

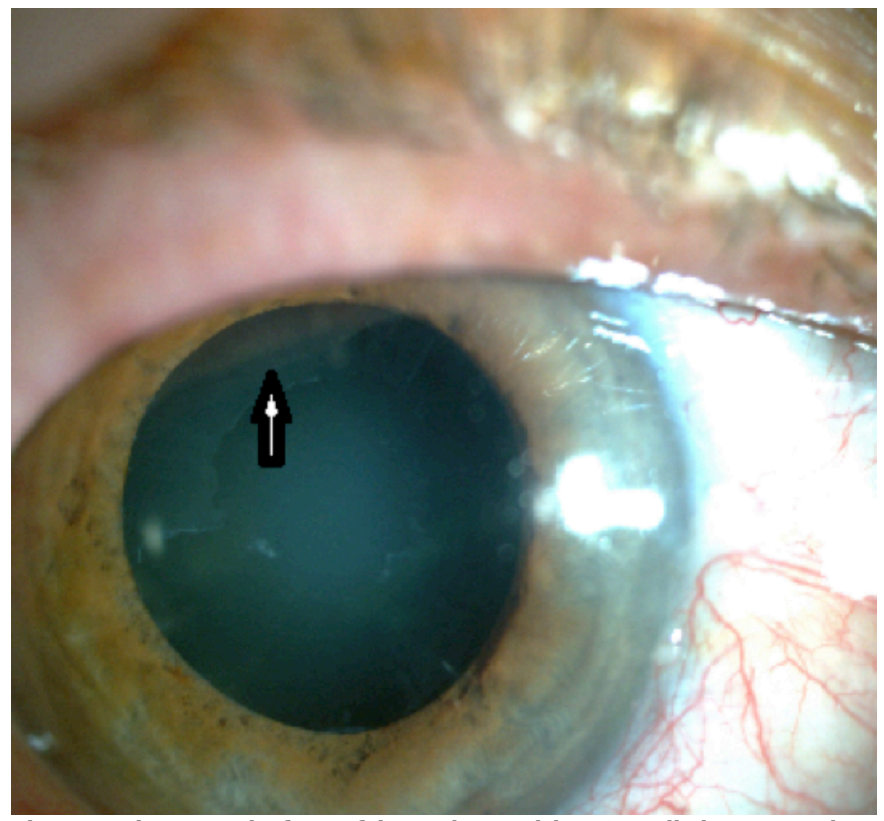

Figure 1: Photograph of one of the patients with XFS on slit-lamp examination. White exfoliation material is shown on the anterior lens capsule.

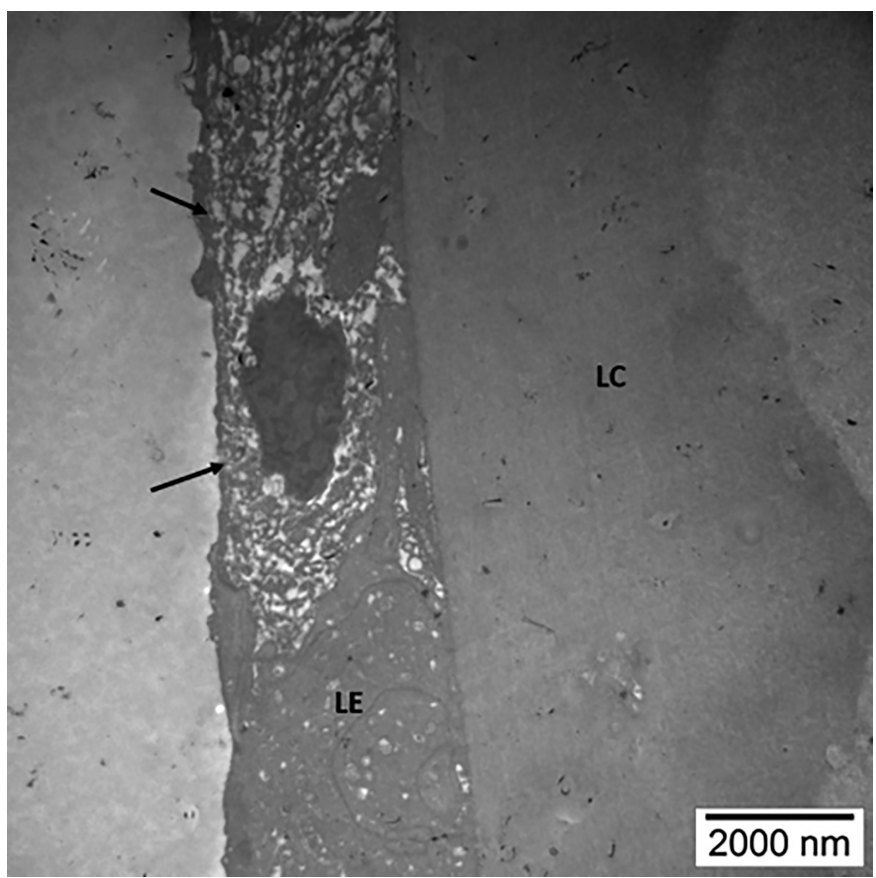

Figure 2: Electron microscopy of the ALC specimen in a patient with XFS showed thinner areas in the lens epithelium (arrows) and vacuolisation $(\times 12,000)$. LC, Lens capsule; LE, lens epithelium.

\section{RESULTS}

In this study, 20 eyes of 20 patients, all of whom underwent phacoemulsification and intraocular lens implantation, were examined. Among those, 10 patients had XFS detected by a slit- 
lamp examination before surgery. The mean age of individuals in the XFS and control groups was $69.4 \pm 6.9(56-82)$ and $65.2 \pm$ 6.9 (54-73) years, respectively. The best-corrected visual acuity in the XFS and control groups was $0.7 \pm 0.4(0.3-1.3)$ logMAR and $0.9 \pm 0.4(0.4-1.3) \log M A R$, respectively. The XFS group had equal numbers of right and left eyes. However, the right eye was affected in four patients and left in six patients in the control group. The demographic and clinical data of the patients included in the study, with and without XFS, are shown in Table I. All the patients in the XFS group demonstrated similar ultrastructural changes in the TEM examination of the ALCs. Notably, the lens epithelium was thinner in the XFS group compared with the control group, which was the most noticeable alteration detected in the XFS group (Figures 2 and 3). Furthermore, thinner areas were detected in the lens epithelium in the XFS group; whereas, no thinning was detected in the lensepithelium in the control group.

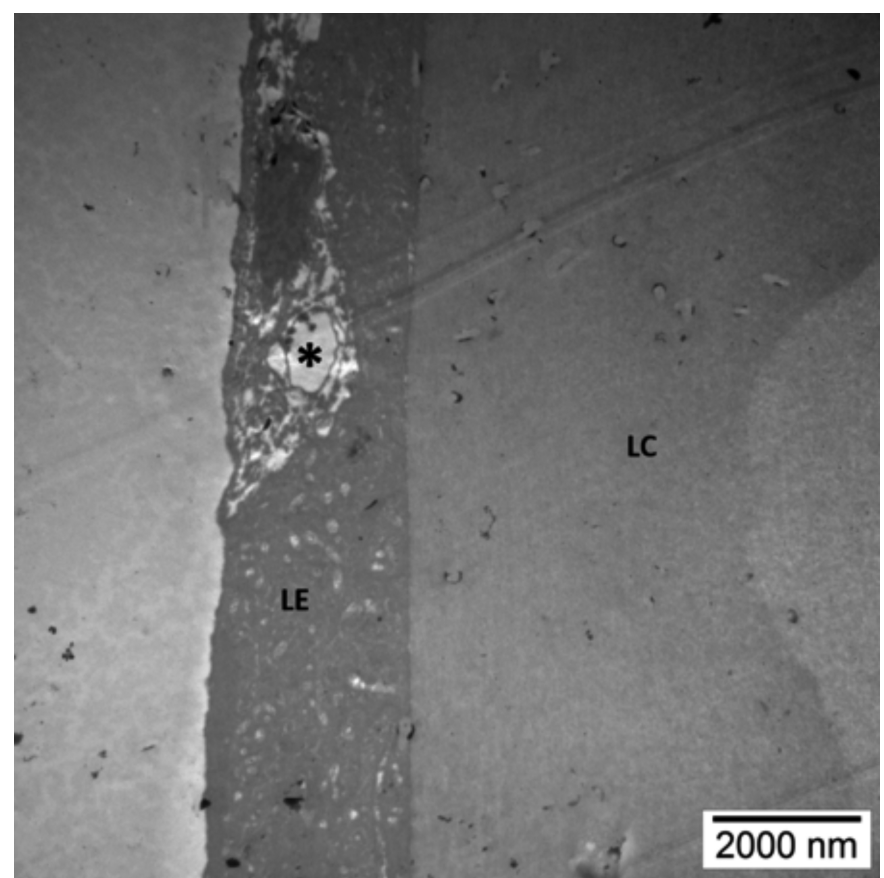

Figure 3: Electron microscopy of the ALC specimen in a patient with XFS showed thinner areas in the lens epithelium and vacuolisation (asterisk) $(\times 12,000)$. LC, Lens capsule; LE, lensepithelium.

Table I: Demographic and clinical data of the patients.

\begin{tabular}{|l|c|c|}
\hline & XFS group & Control group \\
\hline Number of patients $(n)$ & 10 & 10 \\
\hline Mean age (year) (mean \pm SD) & $69.4 \pm 6.9$ & $65.2 \pm 6.9$ \\
& $(56-82)$ & $(54-73)$ \\
\hline Best-corrected visual acuity before & $0.7 \pm 0.4$ & $0.9 \pm 0.4$ \\
surgery (logMAR) & $(0.3-1.3)$ & $(0.4-1.3)$ \\
\hline Type of cataracts & Senile cataracts & Senile cataracts \\
\hline LogMAR, logarithm of the minimum angle of resolution; $n$, number; XFS, exfoliation \\
syndrome.
\end{tabular}

Both squamous and cuboidal cells, as well as increased intracellular vacuolisation, were found in the lens epithelium in the XFS group. Despite ultrastructural changes in the epithelium, the basement membranes were intact with no ultrastructural changes. In the lens epithelial cells, numerous apoptotic cells had pyknotic nuclei and many vacuoles inside the cytoplasm. The sizes of the vacuoles were different in each patient (Figure 3 ). Such ultrastructural changes were not detected in the control group (Figure 4). In the control group, vacuoles were found inside the cytoplasm, but none of the materials were seen in the ALCs.

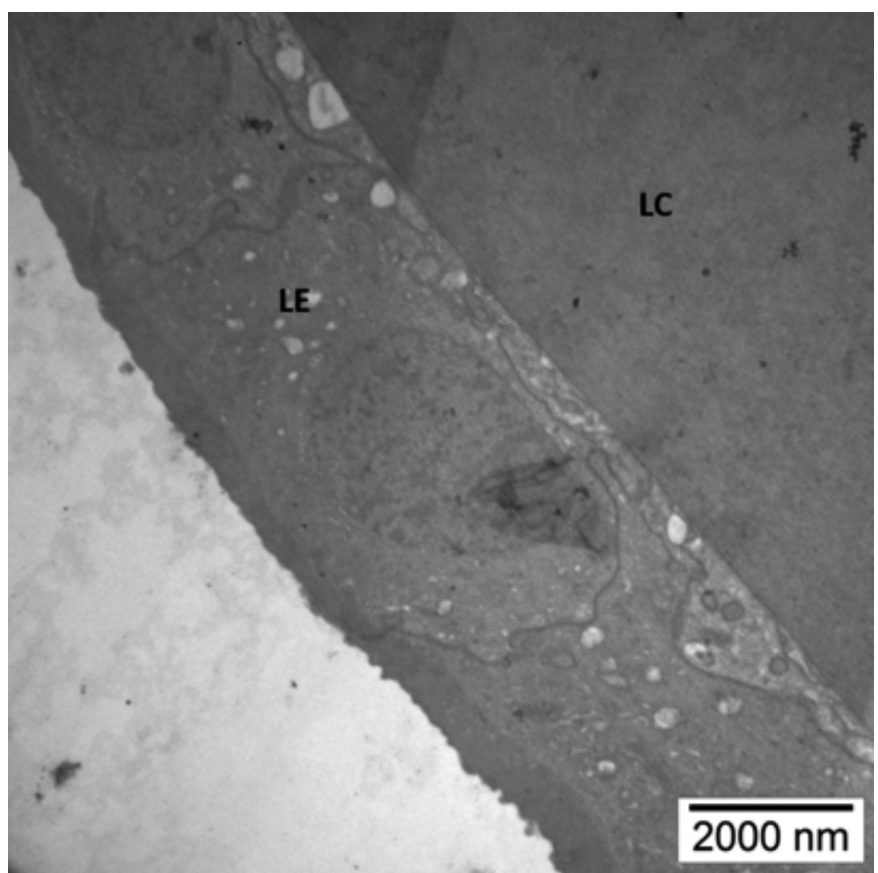

Figure 4: Electron microscopy of the ALC specimen in an individual in the control group with no exfoliative material operated for senile cataracts $(\times 12,000)$. LC, Lens capsule; LE, lens epithelium.

\section{DISCUSSION}

The lens capsule is a modified, transparent basement membrane that completely surrounds the ocular lens. Lens epithelial cells remain undifferentiated under the lens capsule throughout life. ${ }^{8}$ The lens capsule is the thickest basal membrane of the human body; it is produced by the lens epithelium anteriorly and elongated lens fibrils posteriorly. ${ }^{8}$ The lens capsuleappears homogeneous and denseunder the lightmicroscope; whereas, it appears as dense patch-style fibrils parallel to each, especially in the outer parts. ${ }^{8}$ This parallel settlement disappeared with increasing age. ${ }^{8}$ Ultrastructural studies revealed typical exfoliative material deposits on the surface of the basement membrane without any change in the thickness and elasticity of the lens capsule. Sorkou et al. examined ALCs withelectron microscopy and revealed theaccumulation of electron-dense material on the basement membrane surface and lens epithelial cells in patients with pseudoexfoliation syndrome. ${ }^{9}$ This study also showed that some of the exfoliative materials were separated from the epithelium. This material may have been produced by the epithelium, or may be the product of disrupted cells. The content was found to be more intense and homogeneous in regions with pseudoexfoliation accumulation. Sorkou et al. found that the thickness of the basement membrane of the lens epithelium did not change in patients with pseudoexfoliation syndrome, which was in line 
with the findings of this study. ${ }^{10}$ In 1987 , Ruotsalainen and Tarkkanen revealed similar basement membrane thicknesses in both XFS and control groups in the light microscopic examination. ${ }^{11}$ On the contrary, in 1972, Ghosh and Speakman showed that the basement membrane in the eyes with XFS was thicker in some regions of the lens capsule, primarily in the equatorial area. ${ }^{12}$ Additionally, they revealed some detachment areas in the basementmembranes of patients with XFS.

Sorkou et al. revealed epithelial changes in the lenses of patients in the exfoliative group, as in the present study. In addition, they found some detachment areas in the basement membrane and epithelial loss in some parts of the lens. ${ }^{10}$ Further, they demonstrated similar vacuolization and intra-and intercellular edema in the epithelium of the ALCs of patients with pseudoexfoliation, as in the present study. Related studies attempted to explain the differences in degenerative changes detected in ALC with the exfoliative material originating from different places. ${ }^{1,12,13}$

Ultrastructural studies performed on senile cataracts detected intra- and intercellular vacuoles in the lens epithelium and vesicles adjacent to the capsule cells. Patients with senile cataracts without XFS were included in the present study as the control group with the aim of detecting such changes. In their microscopic study, Rahim et al. detected abnormal migration and multilayering of the cells in senile lenses. ${ }^{14}$ Inanç et al. studied the ALCs of patients with traumatic cataracts, all of which showed significant ultrastructural changes compared with that in the control group, namely degenerated lens epithelial cells having variable-sized intraepithelial vacuoles. ${ }^{15}$ In all these studies, the etiopathogenesis of different types of cataracts was detected with the aid of ultrastructural analysis of the lens capsule and lens epithelium under electron microscopy.

This study had several limitations, one of which was the relatively small sample size. However, the number of samples was considered to be adequate because it was an electron microscopic study. This study may pave the way for more detailed future analyses with larger sample sizes. Moreover, the pathophysiologic effects of glaucoma on the lens structure can be detected in more depth with further studies on patients with exfoliative glaucoma.

\section{CONCLUSION}

In conclusion, this study detected some significant ultrastructural changes in patients with XFS in the form of alterations in the lens epithelium with no change in the basement membrane. These changes can help find the origin of the exfoliative material. However, further comprehensive studies are needed to define the structure and the origin of the exfoliative material in detail.

\section{ETHICALAPPROVAL:}

All procedures performed in the studies involving human participants were in accordance with the ethical standards of the Hacettepe University School of Medicine Institutional Review Board and with the 1964 Helsinki Declaration and its later amendments or comparable ethical standards (GO 20/405).

\section{PATIENTS' CONSENT:}

Written informed consent was obtained from the parents of each subject in the study.

\section{CONFLICT OF INTEREST:}

The authors declared no conflict of interest.

\section{AUTHORS' CONTRIBUTION:}

OD: Conception and design, data acquisition and analysis, interpretation, drafting, final approval.

YK: Conception and design, interpretation, critical revision, finalapproval.

AF: Design, data acquisition and analysis, critical revision, final approval.

MFS: Interpretation, data acquisition and analysis, critical revision, final approval.

SK: Interpretation, critical revision, final approval.

\section{REFERENCES}

1. Crandall AS. Exfoliation syndrome and cataract surgery. J Glaucoma 2018; 27 Suppl 1:S102-4. doi: 10.1097/ IJG.000000 0000000925.

2. Asbell PA, Dualan I, Mindel J, Brocks D, Ahmad M, Epstein S. Age-related cataract. Lancet 2005; 365:599-609. doi.10.1016/S0140-6736(05)17911-2.

3. Endres W, Shin YS. Cataract and metabolic disease. J Inherit Metab Dis 1990; 13:509-16. doi: 10.1007/BF0 1799508.

4. Schlötzer-Schrehardt U, von der Mark K, Sakai LY, Naumann GO. Increased extracellular deposition of fibrillincontaining fibrils in pseudoexfoliation syndrome. Invest Ophthalmol Vis Sci 1997; 38(5):970-84.

5. Sargon MF, Celik HH, Orhan M. Electron microscopy of the senile changes in lens epithelium. Okajimas Folia Anat Jpn 1997; 74(2-3):75-9. doi:10.2535/ofaj1936.74.2-3_75.

6. Dark AJ, Streeten BW, Cornwall CC. Pseudoexfoliative disease of the lens: A study in electron microscopy and histochemistry. Br J Ophthalmol 1977; 61(7):462-72. doi: 10.1136/bjo.61.7.462.

7. Puska P, Tarkkanen A. Exfoliation syndrome as a risk factor for cataract development: five-year follow-up of lens opacities in exfoliation syndrome. J Cataract Refract Surg 2001; 27(12):1992-8. doi: 10.1016/s0886-3350(01) 00972-5.

8. Danysh BP, Duncan MK. The lens capsule. Exp Eye Res 2009; 88(2):151-64. doi: 10.1016/j.exer.2008.08.002.

9. Sorkou KN, Manthou ME, Tsaousis KT, Brazitikos P, Tsinopoulos IT. Transmission electron microscopy study of undescribed material at the anterior lens capsule in exfoliation syndrome. Graefes Arch Clin Exp Ophthalmol 2018; 256(9):1631-7. doi: 10.1007/s00417-018-4062-1.

10. Sorkou KN, Manthou ME, Meditskou S, Ziakas N, Tsaousis KT, Tsinopoulos IT. Severe abnormalities of lens epithelial cells in exfoliation syndrome: A transmission electron microscopy study of patients with age-related cataract. Med (Kaunas) 2019; 55(6):235. doi: 10.3390/medicina 
55060235.

11. Ruotsalainen J, Tarkkanen A. Capsule thickness of cataractous lenses with and without exfoliation syndrome. Acta Ophthalmol (Copenh) 1987; 65(4):444-9. doi: 10. 1111/j.1755-3768.1987.tb07021.x.

12. Ghosh M, Speakman JS. Inclusions in human lens capsule and their relationship to senile exfoliation. Can J Ophthalmol 1972; 7(4):413-27.

13. Hammer T, Schlotzer-Schrehardt U, Naumann GO. Unilateral or asymmetric pseudoexfoliation syndrome? An ultrastructural study. Arch Ophthalmol 2001; 119:10231031. doi:10.1001/archopht.119.7.1023.

14. Rahim A, lqbal K. To assess the levels of zinc in serum and changes in the lens of diabetic and senile cataract patients. J Pak Med Assoc 2011; 61(9):853-5.

15. Inanc M, Tekin K, Erol YO, Sargon MF, Koc M, Budakoglu O, et al. The ultrastructural alterations in the lens capsule and epithelium in eyes with traumatic white cataract. Int Ophthalmol 2019; 39(1):47-53. doi: 10.1007/s10792017-0783-0. 